\title{
Transposition
}

Musique et Sciences Sociales

Hors-série 1 | 2018

Musique, histoire, sociétés

\section{Lieux et espaces de la musique}

Retour sur un séminaire

Michael Werner

\section{CpenEdition}

Journals

Édition électronique

URL : http://journals.openedition.org/transposition/1687

DOI : 10.4000/transposition. 1687

ISSN : $2110-6134$

Éditeur

CRAL - Centre de recherche sur les arts et le langage

\section{Référence électronique}

Michael Werner, «Lieux et espaces de la musique », Transposition [En ligne], Hors-série 1 | 2018, mis en ligne le 30 janvier 2018, consulté le 09 février 2020. URL : http://journals.openedition.org/ transposition/1687 ; DOI : 10.4000/transposition.1687

Ce document a été généré automatiquement le 9 février 2020

\section{(c) () ()}

La revue Transposition est mise à disposition selon les termes de la Licence Creative Commons Attribution - Partage dans les Mêmes Conditions 4.0 International. 


\title{
Lieux et espaces de la musique
}

\author{
Retour sur un séminaire
}

\author{
Michael Werner
}

\section{NOTE DE L'AUTEUR}

Ce texte est issu d'une communication au colloque « Arts, littérature et sciences sociales », organisé en juin 2014 dans le cadre des manifestations pour les 40 ans de l'EHESS.

1 Les remarques qui suivent proposent de revenir sur un séminaire de l'École des hautes études, ses préalables et son développement. Ce sera aussi l'occasion d'évoquer quelques questions plus générales touchant aux approches du « faire musique ».

Intitulé, selon les années, «Lieux et espaces de la musique » ou «Espaces et lieux de la musique ", le séminaire a démarré en 2002, parallèlement à la création d'un DEA et d'une formation doctorale "Musique, histoire, société » qui liait l'EHESS comme institution porteuse à l'EPHE, l'ENS et le CNSMDP. Le projet, à la fois scientifique et pédagogique, était directement issu d'un programme de la Fondation européenne de la science sur "Musical Life in Europe, 1600-1900", à l'intérieur duquel Hans-Erich Bödeker (Institut-Max-Planck d'histoire, Göttingen) Patrice Veit et Michael Werner (EHESS) ont codirigé, de 1997 à 2002, un axe portant sur « Le concert et ses publics en Europe, 1700-1920».

3 Sans trop m'appesantir sur les préalables, le déroulement et les résultats de ce projet, qui a réuni historiens, musicologues, historiens d'art, sociologues et géographes pour enquêter sur l'émergence de la "forme concert » et des transformations des pratiques sociales et culturelles qui l'ont accompagnée, je dirais simplement que la question des lieux, plutôt délaissée par la recherche antérieure, est progressivement apparue comme l'un des champs les plus prometteurs, pouvant ouvrir un large éventail de nouvelles pistes d'investigation. Au cours de l'enquête, nous nous sommes naturellement intéressés d'abord aux salles de concert, plus précisément aux constructions dédiées uniquement à l'exécution des concerts, mais, sous l'effet, entre 
autres, du spatial turn qui a affecté les sciences humaines et sociales à la fin des années $1990^{1}$, le champ de vision s'est progressivement élargi à d'autres objets, d'autres échelles et d'autres dimensions de l'espace de la musique ${ }^{2}$. Mais c'était toujours la réalité matérielle de ces lieux et espaces qui a servi de point de départ de l'enquête.

C'est donc sur la base de ces premiers acquis que le séminaire a été lancé en 2002. Coordonné par Patrice Veit et Michael Werner, noyau qu'ont rejoint par la suite Denis Laborde, Karine Le Bail, Claire Guiu et Laura Jouve-Villard, il a été conçu comme un lieu d'accueil et de discussion pour des chercheurs venant d'horizons disciplinaires très divers. Il a, d'emblée, intégré des présentations de travaux de doctorants inscrits dans la formation "Musique, histoire, société » (une bonne douzaine en tout ${ }^{3}$ ), tout en invitant, également dès le début, mais d'une manière de plus en plus conséquente, et grâce notamment aux extraordinaires talents d'entremetteur intellectuel de Patrice Veit, des praticiens et acteurs des lieux musicaux, particularité sur laquelle nous reviendrons au cours de notre propos. Dans l'ensemble, pendant douze années, le séminaire a accueilli quelques 110 conférenciers pour des séances qui, du format classique de deux heures, ont été étendues, à partir de 2006, à celui des demi-journées, pour revenir, en 2013, à celui de trois heures.

Plusieurs lignes de réflexion se dessinent pour résumer et donner une idée, je l'espère, de l'évolution des thématiques et des approches analytiques. Pour simplifier, je propose de les numéroter.

6 1. Tout d'abord, les approches de l'espace se sont considérablement complexifiées. Cela concerne, d'une part, les rapports entre lieux et espaces. Si, dans une représentation géométrique, un lieu peut être considéré comme un point défini par des coordonnées spatiales et se situe donc toujours à l'intérieur d'un plan spatial, les lieux empiriques, qu'ils soient physiques ou sociaux, sont généralement dotés d'un double rapport à l'espace. Ils possèdent leur propre organisation spatiale (qu'on pourrait dire interne), tout en s'inscrivant, en même temps, dans un espace environnant (qu'on pourrait dire externe), dont les dimensions varient selon la focale (ou l'échelle) adoptée. Mais ces deux rapports à l'espace ne sont pas indépendants l'un de l'autre. La spatialité interne du lieu entretient de multiples relations avec l'espace extérieur. Et celles-ci ne passent pas seulement par les ouvertures spécialement aménagées pour organiser les flux, les allers et venues, mais aussi par les événements et programmes qui s'y déroulent, ainsi que, surtout, par les actions des personnes qui circulent dans les deux espaces. Cela a été développé, sur le plan historique, à propos du théâtre du Châtelet (Gesa zur Nieden) ou le Teatro di San Carlo de Naples (Mélanie Traversier), mais a également été une préoccupation constante pour l'étude des salles contemporaines lancée au fil des années. D'autre part, la prise en compte de la dimension symbolique des espaces, plus précisément de la manière dont les usages des espaces physiques se chargent de représentations normatives et créent des ordres immatériels, a attiré l'attention sur d'autres questions à travailler. Ici aussi, ce n'est pas tant la distinction entre niveau matériel et niveau symbolique qui est à explorer, mais les interrelations nombreuses qui, en liant les deux, participent à leur construction réciproque. Architectes, acousticiens, planificateurs urbains, musiciens, du compositeur à l'instrumentiste, et usagers de toute sorte concourent afin de faire exister la musique dans des configurations spatiales spécifiques. Pour démêler cet écheveau, la rencontre avec le spatial turn a été d'une importance capitale. En envisageant l'espace non plus comme un déjà-là, un simple contenant, mais comme un ensemble dynamique produit par 
l'interdépendance des dimensions matérielles, sociales et symboliques de l'espace, les perspectives ouvertes par la nouvelle sociologie de l'espace ${ }^{4}$ nous ont accompagnés pour investir, à nouveaux frais, des terrains d'investigation traditionnels comme l'espace sonore, ou encore peu travaillés, comme la distribution spatiale d'un festival (déployée par Joséphine Markovits) ou la topographie d'une pratique musicale conduite par des exilés (Anna Langenbruch).

7 2. Cette extension thématique s'est accompagnée d'une ouverture de plus en plus marquée à des intervenants acteurs de la vie musicale, en premier lieu les compositeurs. Les premiers parmi eux qui sont venus animer des séances, notamment Pascal Zavaro et François Bayle, ont introduit des conceptions de l'espace où les frontières entre distribution matérielle des sources sonores et construction de l'espace d'écoute sont devenues instables. Par la suite, Mark André (qui est intervenu à deux reprises) a développé comment des représentations spatiales et des formes de "placement» ont pu structurer des processus de composition, si bien que l'œuvre apparaît comme une construction dramaturgique déterminée par des relations spatiales entre les entités qui la composent. Isabel Mundry, de son côté, s'est interrogée sur la structuration de l'œuvre musicale qu'elle assimile à un tissu de lignes qui se croisent, tant au niveau de la composition qu'à celui de l'écoute. Ainsi, l'œuvre est pour elle un « lieu » fait de relations spatiales qui s'inscrivent également dans une dimension temporelle. Établissant un parallèle à Cézanne (pour la peinture) et Péter Nádas (pour la littérature), elle insiste sur les tensions induites, lors de l'écoute, par la double perception de l'espace et du temps. Jean-Luc Hervé a présenté son œuvre "Germination» qui est en même temps une installation urbaine, Pascale Criton a évoqué sa conception des espaces tonaux.

8 3. Au fil des années, les rencontres avec les praticiens se sont multipliées. Du côté des organisateurs de musique, des architectes et concepteurs de salles ou encore des acousticiens, nous avons eu la chance d'accueillir Laurent Bayle, Joséphine Markovits, Peter Androsch (qui est aussi compositeur), Thomas Bleek, Brigitte Métra, Xavier Fabre, Gaspard Joly, Bruno Suner, Christine Simonin, Yann Rocher, Cécile Régnault, Alex Arteaga, Jean-Paul Lamoureux, Jean-Michel Fayez ou encore Marc Quiquerez et Fumiaki Salamaki de l'agence Nagata/Toyota, entre autres. Chacune de ces rencontres a été l'occasion d'une plongée dans un projet concret, une programmation ainsi que d'une discussion sur un retour d'expérience. Parmi les musiciens qui sont venus évoquer leur rapport à l'espace du son, on peut faire une référence particulière à Mireille Delunsch qui a analysé l'espace sonore corporel tel qu'il est ressenti et travaillé par l'artiste lyrique ou à Marina Chiche qui a développé, exercices pratiques à l'appui, non seulement sa vision du rapport entre le son du violon et le corps de l'instrumentiste, mais aussi celle des transformations que la sonorité du violon a vécues au cours des derniers siècles ainsi que des effets de ces transformations sur le jeu et le répertoire. Malcolm King, de son côté, est venu discuter son approche de l'espace de la voix tant du point de vue physiologique et de la production du son que de celui de la projection sur une scène ou dans une salle. Winfried Bönig, enfin, a exposé le cas particulier de l'orgue et de son insertion dans l'espace de l'église qui fait corps avec la sonorité de l'instrument. D'autres types de praticiens se sont prêtés à l'exercice : des ingénieurs du son (François Eckert, Jean-Marc Lydzwa, Jean Umansky), scénographes (Jean-Michel Fayet, Laurent Gachet), metteurs en ondes (Daniel Zalay), spécialistes de psychoacoustique (Claude Bailblé) ou encore des responsables d'actions culturelles ou de projets urbains (Antoine Naso, Geoffroy Vauthier, Stella Sainson, Kamel Dafri) et des 
urbanistes (Olivier Soubeyran, Marie-Hélène Massot). Souvent d'ailleurs, ces acteurspraticiens cumulent plusieurs catégories socioprofessionnelles, se trouvent au croisement de tout un ensemble d'activités et de métiers. L'une des caractéristiques de ces rencontres a été qu'elles ont permis de dépasser le clivage, souvent mis en avant, entre chercheurs et praticiens ou créateurs. Les échanges ont non seulement produit un enrichissement réciproque des questionnaires, mais aussi provoqué un retour réflexif sur les approches et les points de vue. Les frontières entre théorie et pratique, réflexion épistémologique et action sur les différents terrains se sont déplacées. Elles ne sont plus apparues comme un préalable, mais comme un gros faisceau de fils enchevêtrés traversant une multitude de problématiques et de modalités d'action. La qualité de la réflexion proposée par nombre de ces " praticiens » n'a strictement rien à envier à celle des chercheurs. Et la posture d'objectivation systématique réclamée naguère par le chercheur en sciences sociales ne résiste plus à l'épreuve des savoirs, à la fois théoriques et pratiques, accumulés par les acteurs du terrain. Du coup, le retour réflexif sur les propres pratiques, aussi bien du côté du chercheur que de celui des acteurs de la vie musicale, s'est trouvé constamment enrichi par l'intégration du point de vue de « l'autre » et la mise en relation des positionnements respectifs.

4. À partir de 2008 et sous l'impulsion notamment de Karine Le Bail et de Claire Guiu qui avaient successivement rejoint l'équipe, certains lieux de musique ont fait l'objet d'investigations plus poussées. C'était le cas de deux grands chantiers, celui de la rénovation du Grand Auditorium de la Maison de la Radio et celui de la Philharmonie de Paris. Conséquence : toute une série de séances « hors les murs", sur le terrain, à la Maison de la Radio, à la Villette, à Pleyel, à l'opéra Bastille. Le chantier de la Philharmonie à la Villette, notamment, a déployé une force d'attraction irrésistible qui nous a entraînés in situ afin de nous confronter, concrètement, aux problèmes posés par la construction d'un lieu de musique prestigieux. Ces démarches ont été, de 2010 à 2012, couplées aux activités de deux classes de Master des écoles d'architecture de Paris-Belleville et de Paris-Malaquais sous la direction de Christine Simonin et de Yann Rocher, auxquelles se sont associés, pour une enquête sur l'accessibilité de la future Philharmonie, des étudiants de l'Ecole d'architecture de Lyon (ENSAL). Geoffroy Vauthier, ingénieur responsable de la maîtrise d'ouvrage (et, par ailleurs, musicien pianiste), a accueilli à plusieurs reprises le séminaire chez lui sur le chantier de la Villette, tout en ne dédaignant pas de faire un détour dans les salles de séminaire pour venir écouter, entre autres, un travail de restitution collective, présenté par des étudiants, sur les questions d'accessibilité de l'emplacement Porte de Pantin. Parallèlement, les études sur le terrain de la Philharmonie se sont élargies par un programme de recherche et de formation à la recherche franco-allemande du CIERA qui, sous l'égide notamment de Claire Guiu, Karine Le Bail, Yann Rocher et Patrice Veit, a réuni entre 2011 et 2013 le Centre Georg Simmel, le Centre Marc Bloch de Berlin, l'Université technique de Berlin et l'Ecole d'architecture de Paris-Malaquais dans un projet d'analyse croisée des lieux scéniques à Berlin et à Paris. L'atelier de recherche d'une semaine qui a eu lieu dans ce cadre à Paris en novembre 2012 sur le thème « Un lieu de musique réinvente-t-il la ville ? " a fourni aux étudiants du séminaire l'occasion d'une plongée passionnante dans le processus de remodelage du paysage musical urbain de Paris, devant l'arrière-plan de ce qui s'est construit à cet égard à Berlin pendant les dernières décennies. Enfin, par l'intermédiaire de Damien Verger et Denis Laborde, les participants du séminaire ont eu l'occasion, en 2013, de se familiariser avec le projet DEMOS (Dispositif d'éducation musicale et orchestrale à vocation sociale) qui, 
sur le site de la Cité de la musique associée à la future Philharmonie de Paris, vise à démocratiser la formation musicale par l'apprentissage en orchestre chez les jeunes des quartiers défavorisés de la proche banlieue Nord-Est.

10 5. Le séminaire a également élargi le périmètre géographique et les cadrages normatifs des lieux explorés. Si les théâtres et salles à Naples, Milan, Vienne, Londres, Amsterdam, Bruxelles, Versailles, Paris, Berlin, Cologne ou Leipzig ont fait dès le départ, en tant que lieux consacrés, l'objet d'analyses détaillées, des endroits plus insolites et des réalisations plus récentes sont bientôt entrés dans le champ de vision. Mentionnons, à titre d'exemple, la Casa da musica de Porto (présenté par Nino Grande), des salles dites "polyvalentes" en région (Brigitte Métra), le réaménagement des friches industrielles de la Ruhr en lieu de fabrication d'espaces sonores (Tobias Bleek), les théâtres utopiques sous la République de Weimar (Yann Rocher), les expériences d' Auditive Architecture menées à l'Université technique de Berlin (Alex Arteaga), la transformation d'une ancienne prison en maison de la culture à Florence (Rémi Wagogne), la reconversion d'un coron en lieux de musique à Oignies appelé "Métaphone " (Isabel Hérault), la composition d'un territoire musical dans le parc naturel du Haut-Jura (Cécile Regnault), les coulisses du « théâtrophone » qui a fasciné le public parisien dans les années 1880 (Melissa Vand Drie), le métro parisien et sa "programmation » musicale pendant les deux dernières décennies (Antoine Naso et Stella Sainson) ou encore les sous-sols de la Conciergerie à Paris comme lieu d'expérimentation d'acoustique par le toucher (Pascale Criton). D'autres séances nous ont transportés plus loin, à la Praça Once de Rio de Janeiro (Laura Jouve-Villard), dans les quartiers «tango » de Buenos Aires (Elsa Broclain), au nord du Mali pour le festival du Désert (Marta Amico) ou dans les contreforts des Apennins vers l'Auditorium del parco conçu par Renzo Piano à l'Acquila. Mais chaque fois, espace matériel et espace représenté se sont fondus dans un objet d'investigation stimulant, requérant des approches analytiques croisées et des pratiques d'interdisciplinarité renouvelées.

11 6. La thématique centrée sur les lieux et espaces a également conduit à un travail sur les catégories non seulement d'«espace» et de «lieu» mais aussi celles relevant d'autres types de questions. Pour donner un exemple, on peut citer l'opposition (en elle-même problématique) entre musique savante et musique populaire, car elle traverse l'ensemble des genres et dépasse les différentes catégorisations, qu'elles viennent des musicologues, de la critique musicale ou des opérateurs du marché de la musique. À côté de la musique classique, ancienne et contemporaine, nous nous sommes penchés sur le jazz (Wenceslas Lizé), le rap (A. Pecqueux), les musiques du monde dans leurs différentes déclinaisons (Denis Laborde, Talia Bachir-Loopuyt, Laura Jouve-Villard) ainsi que les aménagements et installations sonores (Claire Guiu, JeanPaul Thibault, Christine Simonin). D'autres questions ont été posées à propos de processus de labellisation comme «villes créatives» (Elsa Vivant), "musiques nomades » (Antoine Pecqueux, Marta Amico), «Hörstadt » (Peter Androsch) ou encore de notions comme « sound space » ou « paysage sonore » développées lors de plusieurs séances par Claire Guiu. Dans tous ces cas, il a fallu tenir compte du fait qu'il s'agit à la fois de catégories analytiques convoquées, de façon plus ou moins réflexive, par le chercheur et de catégories d'action mobilisées par les acteurs. Ces confrontations ont également mis en évidence l'apport des approches réélaborées de la géographie sociale et culturelle et des études urbaines qui, au-delà du carré existant des historiens, sociologues, anthropologues et musicologues au départ du projet, eux-mêmes en quête 
de pistes nouvelles, ont contribué à leur tour à élargir considérablement notre horizon de questionnement.

12 7. L'un des terrains abordés, pour ainsi dire de côté, a été l'histoire de l'écoute musicale. En dépit des travaux pionniers de James H. Johnson, Peter Gay, William Weber, Peter Szendy et Martin Kaltenecker ${ }^{5}$ (les trois derniers ont été accueillis au séminaire), ce domaine manque toujours de fondations solides et unanimement reconnues, principalement en raison de la rareté des sources empiriques directes, mais sans doute également à cause de la complexité de l'ouïe, l'appareil sensitif impliqué. Le problème de la modélisation de l'écoute qui viserait à distinguer les différentes étapes du processus de "donner signification" au fil de la perception auditive semble effectivement plus ardu encore que pour le visuel. Aux deux dimensions de la perception visuelle (il est vrai retraduite, par le cerveau, en représentation à trois axes) s'ajoute en effet, ici et immédiatement, une troisième. Compilant les informations venant des deux oreilles, l'espace de l'audition est d'emblée tridimensionnel. Du coup, les systèmes de différenciation par lesquels l'audition opère pour interpréter les signaux sonores sont difficiles à démêler. Et en plus, les signaux eux-mêmes peuvent à leur tour être d'une complexité extrême. Dans le cas d'une musique symphonique par exemple, les signaux acoustiques sont générés par un vaste éventail d'instruments aux attributs sonores particuliers qui font évoluer la masse sonore en se combinant incessamment entre eux, dans un double jeu de distinction et de fusion. Enfin tout est lié ici à la qualification de masses de sons dans le temps, avec des caractéristiques qualifiantes (tempo, intensité, niveau, amplitude, fréquence, timbre, etc.), et des dynamiques propres induites par les transformations successives de toutes ces caractéristiques à travers le déroulement temporel de la musique. L'un des défis analytiques est donc qu'il ne suffit pas d'isoler les caractéristiques des constituants sonores, mais que ceux-ci produisent leurs effets à travers d'innombrables combinaisons. La tentative d'élaborer un modèle régulateur de l'écoute fondé sur des différenciations superposées et des réagrégations de traits séparés dans l'analyse se heurte à des obstacles sérieux.

L'affaire se complique, en outre, par trois difficultés supplémentaires. La première tient au fait que, dans l'appareil de perception, l'ouïe est plus directement que l'œil connectée au domaine du sensible et déclenche plus immédiatement des réactions qui sont de l'ordre de l'émotion. Cette charge émotionnelle peut infléchir et rendre plus opaque le travail de différenciation des signaux acoustiques. La deuxième difficulté concerne l'intervention de la mémoire dans le traitement de la perception acoustique. Chacun sait d'expérience que la première écoute d'une musique se distingue assez nettement des écoutes suivantes. À la réécoute interviennent les empreintes des écoutes antérieures, mobilisant les registres, à la fois contextualisants, sensibles et analytiques de la mémoire. Tout comme l'odorat, l'ouïe fonctionne dans une interaction forte avec les expériences stockées qui sont utilisées pour la re-sémantisation, analytique ou émotionnelle, de la perception. Du coup, des dispositifs analytiques de l'écoute fondés sur la seule perception sont insuffisants. Une dernière difficulté est que, dans une situation d'écoute concrète, par exemple lors d'un concert, la perception auditive est en général couplée à des impressions visuelles. Les "mises en scène " visuelles dans la salle de concert ont toujours joué un rôle considérable pour la réception de la musique. Le phénomène est encore bien plus accentué dans des clips 
musicaux ou des chorégraphies s'appuyant sur des techniques vidéo de plus en plus sophistiquées.

On le voit: le thème des espaces et lieux de musique a sans doute ouvert plus de questions que trouvé des réponses. Côté "output», il faut toutefois mentionner les thèses de doctorat dont il a inspiré le sujet. Celles d'Étienne Jardin, Gesa zur Nieden, Anna Langenbruch, Talia Bachir-Loopuyt, Marta Amico, Fanny Gribenski et Laura Jouve-Villard ont, chacune en adoptant une perspective spécifique, contribué à éclairer des coins du vaste terrain de recherche que nous avons essayé d'arpenter. La très grande majorité des doctorants passés par ce lieu d'échange est aujourd'hui en poste dans des universités ou institutions françaises et étrangères, affiliés à des disciplines variées, de l'histoire et de la musicologie à la sociologie et l'ethnologie, l'architecture et les études littéraires. Quatre doctorantes ont, par ailleurs, organisé un colloque junior dont les résultats ont donné lieu à un livre remarquable et remarqué ${ }^{6}$. Le séminaire sur les lieux est devenu lui-même un lieu, structurant, à travers sa double logique de recherche et de formation à la recherche, à travers ses frontières ouvertes, ses formes de discussion et sa curiosité virale, le lieu de sociabilité intellectuelle d'une petite communauté de chercheurs.

Paradoxalement, ce petit retour en arrière a dû faire l'impasse sur les contributions aussi nombreuses que précieuses des chercheurs qui ont animé, par leurs présentations, les différentes séances au fil des années et pour lesquels ce type d'intervention appartenait au quotidien de la vie des séminaires. Ce n'est pas seulement le manque de place, pourtant la raison essentielle de ce renoncement, qui est ici en question. L'orientation même de cet essai de compte rendu qui s'est proposé d'insister sur les décentrages, les dépaysements et les passages de frontières voulait qu'il ne faille pas trop s'attarder sur le fonds de commerce ordinaire de l'exercice. Ce n'est pas minimiser leurs apports, absolument essentiels pour que le dialogue, ou plutôt le " polylogue » ait pu s'établir. Et bien entendu, je n'ai fait ici qu'essayer de retranscrire quelques points forts d'une expérience collective qui, tout en étant animée par une petite équipe d'organisateurs dont je ne suis ici que le simple porte-parole, ne pouvait se réaliser que grâce au concours de tous les participants, intervenants ou discutants venant d'horizons variés. C'est eux qui ont fait vivre ce lieu et la dette que nous avons à leur égard est aussi immense qu'ineffaçable.

\section{BIBLIOGRAPHIE}

BACHIR-LOOPUYT, Talia, IGLESIAS, Sara, LANGENBRUCH, Anna et NIEDEN, Gesa zur (eds), Musik, Kontext, Wissenschaft. Interdisziplinäre Forschung zu Musik / Musique, contextes, savoirs. Perspectives interdisciplinaires sur la musique, Frankfurt, Peter Lang, 2012. 
BACHMANN-MEDICK, Doris, « Spatial Turn », Cultural Turns. Neuorientierungen in den Kulturwissenschaften, $3^{\mathrm{e}}$ édition, Reinbek, Rohwolt, 2009, p. 284-328.

BACHMANN-MEDICK, Doris, LIPPUNER, Roland et LOSSAU, Julia, « In der Raumfalle. Eine Kritik des spatial turn in den Sozialwissenschaften », Soziale Räume und kulturelle Praktiken. Über den strategischen Gebrauch von Medien, sous la direction de Georg MEIN et Markus RIEGER-LADICH, Bielefeld, Aisthesis, 2004, p. 47-64.

BELINA, Bernd et MICHEL, Boris (eds), Raumproduktionen. Beiträge der Radical Geography, Münster, Westfälisches Dampfboot, 2008.

BÖDEKER, Hans-Erich, VEIT, Patrice et WERNER, Michael (eds), Espaces et lieux de concert en Europe 1700-1920. Architecture, musique, société, Berlin, Berliner Wissenschaftsverlag, 2008.

DÜNNE, Jörg et GÜNZEL, Stephan (eds), Raumtheorie. Grundlagentexte aus Philosophie und Kulturwissenschaften, Frankfurt a. M., Suhrkamp, 2006.

GAY, Peter, The Naked Heart. Bourgeois Experience IV, New York, W.W.Norton, 1995

JOHNSON, James H., Listening in Paris. A Cultural History, Los Angeles, University of California Press, 1995.

KALTENECKER, Martin, L'oreille divisée. Les discours sur l'écoute musicale aux XVIII et XIX ${ }^{e}$ siècles, Paris, Musica Falsa, 2010.

LÖW, Martina, «The Constitution of Space. The Structuration of Spaces through the Simultaneity of Effect and Perception », European Journal of Social Theory 11/1 (2008), p. 25-49.

LÖW, Martina, Raumsoziologie, Frankfurt, Suhrkamp, 2001 ; traduction française sous le titre Sociologie de l'espace, Paris, Éditions de la Maison des sciences de l'homme, 2016.

SCHRÖDER, Iris, Das Wissen von der ganzen Welt. Globale Geographien und räumliche Ordnungen Afrikas und Europas 1790-1870, Paderborn, Schöningh, 2011.

SZENDY, Peter, Écoute. Une histoire de nos oreilles, Paris, Éditions de Minuit, 2001

WEBER, William, « Did People Listen in the Eighteenth Century? », Early Music 25 (1997) numéro spécial « Listening Practice », p. 678-691.

\section{NOTES}

1. La littérature sur le spatial turn est abondante. Citons simplement BACHMANN-MEDICK, Doris, LIPPUNER, Roland et LOSSAU, Julia, «In der Raumfalle. Eine Kritik des spatial turn in den Sozialwissenschaften ", Soziale Räume und kulturelle Praktiken. Über den strategischen Gebrauch von Medien, sous la direction de Georg MEIN et Markus RIEGER-LADICH, Bielefeld, Aisthesis, 2004, p. 47-64 ; DÜNNE, Jörg et GÜNZEL, Stephan (eds), Raumtheorie. Grundlagentexte aus Philosophie und Kulturwissenschaften, Frankfurt a. M., Suhrkamp, 2006 ; BACHMANN-MEDICK, Doris, "Spatial Turn ", Cultural Turns. Neuorientierungen in den Kulturwissenschaften, $3^{\mathrm{e}}$ édition, Reinbek, Rohwolt, 2009, p. 284-328 ; BELINA, Bernd et MICHEL, Boris (eds), Raumproduktionen. Beiträge der Radical Geography, Münster, Westfälisches Dampfboot, 2008 ; SCHRÖDER, Iris, Das Wissen von der ganzen Welt. Globale Geographien und räumliche Ordnungen Afrikas und Europas 1790-1870, Paderborn, Schöningh, 2011; LÖW, Martina, "The Constitution of Space. The Structuration of Spaces through the Simultaneity of Effect and Perception », European Journal of Social Theory 11/1 (2008), p. 25-49. 
2. Les travaux relatifs aux lieux et espaces du concert émanant du groupe de l'ESF sur le concert ont été réunis dans le volume BÖDEKER, Hans-Erich, VEIT, Patrice et WERNER, Michael (eds), Espaces et lieux de concert en Europe 1700-1920. Architecture, musique, société, Berlin, Berliner Wissenschaftsverlag, 2008.

3. Étienne Jardin, Gesa zur Nieden, Yann Rocher, Anna Langenbruch, Talia Bachir, Sara Iglesias, Damien Verger, Laura-Jouve Villard, Fanny Gribenski, Marta Amico, Florine Andrieux, Sophie Picard, Julien Ségol, Elsa Broclain, auxquels se sont ajoutés des doctorants venant d'autres écoles et formations doctorales.

4. Voir en particulier LÖW, Martina, Raumsoziologie, Frankfurt, Suhrkamp, 2001; traduction française sous le titre Sociologie de l'espace, Paris, Éditions de la Maison des sciences de l'homme, 2016.

5. Juste pour mémoire: JOHNSON, James H., Listening in Paris. A Cultural History, Los Angeles, University of California Press, 1995 ; GAY, Peter, The Naked Heart. Bourgeois Experience IV, New York, W.W.Norton, 1995 ; WEBER, William, « Did People Listen in the Eighteenth Century? », Early Music 25 (1997) numéro spécial «Listening Practice», p. 678-91; SZENDY, Peter, Écoute. Une histoire de nos oreilles, Paris, Éditions de Minuit, 2001 ; KALTENECKER, Martin, L'oreille divisée. Les discours sur l'écoute musicale aux XVIII et XIX ${ }^{e}$ siècles, Paris, Musica Falsa, 2010.

6. BACHIR-LOOPUYT, Talia, IGLESIAS, Sara, LANGENBRUCH, Anna et NIEDEN, Gesa zur (eds), Musik, Kontext, Wissenschaft. Interdisziplinäre Forschung zu Musik / Musique, contextes, savoirs. Perspectives interdisciplinaires sur la musique, Frankfurt, Peter Lang, 2012.

\section{RÉSUMÉS}

L'article propose un retour réflexif sur le séminaire «Lieux et espaces de la musique » qui s'est tenu à l'EHESS de 2002 à 2014. Lancé par Patrice Veit et Michael Werner, auxquels se sont joints, par la suite, Denis Laborde, Karine Le Bail, Claire Guiu et Laura Jouve-Villard, ce séminaire au format de séances longues (trois à quatre heures) a été un lieu d'expérimentation et de rencontre. Réunissant environ 120 intervenants et conférenciers autour de questions liées à la spatialité, matérielle et symbolique, de l'activité musicale et de son inscription dans le social, il a eu la particularité d'associer chercheurs et acteurs-praticiens, du compositeur à l'ingénieur du son, en passant par les musiciens, architectes, urbanistes, scénographes, metteurs en onde, acousticiens et responsables de programmation culturelle. Tout en tentant de redessiner les étapes de l'avancement de la réflexion collective, l'article évoque également quelques thématiques transversales qui ont accompagné la discussion. On peut citer la question des catégories d'analyse et d'action propres à la dimension spatiale, les problèmes de la perception auditive et de l'écoute ou encore les relations complexes entre action individuelle et structuration des différents collectifs. Enfin on insiste sur l'aspect formation du séminaire qui valait non seulement pour les étudiants, mais aussi pour les chercheurs, qu'ils aient été intervenant, discutant ou coordinateur. 


\section{INDEX}

Mots-clés : lieux et espaces de la musique, musique et espace, histoire des pratiques musicales, histoire et sociologie de l'écoute musicale, anthropologie de la musique, interdisciplinarité, villes créatives, arts et sciences, architecture musicale, acoustique musicale

\section{AUTEUR}

\section{MICHAEL WERNER}

Michael Werner (Centre Georg Simmel, EHESS) est historien de la culture. Il a fait émerger, avec Michel Espagne, le champ de recherche des transferts culturels et problématisé, avec Bénédicte Zimmermann, les approches relationnelles en histoire et en sciences sociales en proposant les outils de l'histoire croisée. Sur le plan empirique, ses terrains de recherche ont été, entre autres, l'histoire des disciplines de sciences humaines et sociales, les pratiques culturelles de la musique, l'histoire des émigrés allemands en France (avec une place particulière à Heinrich Heine) ainsi que, plus largement, l'histoire franco-allemande du XVIII ${ }^{\mathrm{e}}$ au Xx ${ }^{\mathrm{e}}$ siècles.

Auteur de 'Der Zweck des Lebens ist das Leben selbst'. Heinrich Heine, eine Biographie (avec JanChristoph Hauschild, 2006 [1997]) et co-éditeur de De la comparaison à l'histoire croisée (avec Bénédicte Zimmermann, 2004), Espaces et lieux de concert en Europe 1700-1920. Architecture, musique, société (avec Hans Erich Bödeker et Patrice Veit, 2008), Multiple Antiquities, Multiple Modernities. Ancient Histories in Nineteenth Century European Cultures (avec Gábor Klaniczay et Ottó Gecser, 2011), il a récemment publié « Les débuts de la fondation Royaumont dans le contexte des fondations philanthropiques internationales ", LABORDE, Denis (ed), Le cas Royaumont, Grâce, Éditions Créaphis, 2014 ; et « Comprendre l'action musicienne. Remarques sur les transformations des mondes de la musique au XIX siècle », LILTI, Antoine, LORIGA, Sabina, SCHAUB, Jean-Frédéric et SEBASTIANI, Silvia (eds), L'expérience historiographique, Paris, Éditions de l'EHESS, 2016. 